\title{
Overcoming Singularity of Euler Angles in Robot Pose Estimation using Axis Rotation
}

\author{
Mohammad Ehab Ragab \\ The Electronics Research Institute \\ Tahrir Street, Dokki, \\ Giza, Egypt
}

\begin{abstract}
In this paper, the singularity of Euler angles rotation representation in robot pose estimation is overcome. This is accomplished through coordinate system rotating and signadjusting of the intrinsic parameter camera matrices. A stereo pair is attached to the robot and the extended Kalman filter is used as a recursive pose estimator. An extensive set of synthetic and real experiments have been carried out under various motion patterns in both singular and nonsingular settings. The approach has proved accurate in face of singularity and stable during Jacobian calculations as well.
\end{abstract}

\section{General Terms}

Computer Vision, Robotics.

\section{Keywords}

Pose Estimation, Stereo, Euler Angles, Extended Kalman Filter.

\section{INTRODUCTION}

Pose estimation is a classic problem in various engineering fields. To solve this problem, the location and orientation (rotation) of objects should be obtained. Many applications require solving this problem. For example, in [1] the location and speed of a vehicle are communicated in case of an accident. Pose estimation is used in [2] for optimizing the turning velocity of a robot. Unmanned aerial vehicle pose estimation is performed in [3] for live sports production. The three-dimensional model-based tracking of a tennis court is obtained using a monocular camera.Using computer vision in pose estimation is advantageous especially indoors where the global positioning system (GPS) is denied. Moreover, computer vision systems which use ordinary cameras as their sensors are less intrusive than most of the other systems. Additionally, they do not suffer from drift such as odometric systems [4].

To solve the pose estimation problem in real time (e.g. for a mobile vehicle), there is a need to adopt a recursive technique such as the extended Kalman filter (EKF) [5], [6], and [7]. The pose parameters (rotational and translational) are encoded in the state space vector of the EKF. Although there are various representations of the rotation, the Euler angles are more tangible. They describe the rotation angles around the chosen coordinate system axes. The first advantage of using them is that the axes are selected to be attached to a physical entity such as a reference camera or a mobile platform. This makes the Euler angles both meaningful and measurable. The second advantage is referring the rotation to the same axes used to measure the translation. However, the Euler angles representation of rotation suffers from certain singularities which will be described in section 2 below. These singularities are even aggravated when calculating the derivatives inside the EKF Jacobian. There are other rotation representations which do not suffer from singularities such as quaternions and axis- angle. However, such representations do not offer the direct physical interpretation as Euler angles. Additionally, they are not minimal compared to Euler angles which use only three angles to describe the three degrees of freedom of the rotation. Moreover, they are not one-to-one mappings [5], and [8].In this work, the singularity of Euler angles rotation representation is overcome using axis rotation. This is particularly inspired by the nature of robot motion indoors or outdoors on nearly even surfaces. Accordingly, the robot holds an upright posture with hardly any rotation around the axes parallel to the ground plane. A stereo pair is used for obtaining the three dimensional (3D) structure, and for outlier feature rejection. Since the robot pose is estimated in real time, the EKF is utilized as a recursive estimator.

The rest of this paper is organized as follows: the camera utilization, the EKF implementation, and the suggested rotation of coordinate axes are described in section 2 . The simulation and real experiments are shown in section 3 while the results are discussed in section 4 . The paper is concluded in section 5 .

\section{METHOD}

\subsection{EKF Implementation and Euler Angles}

The pose EKF has a state space vector composed of six pose parameters and their derivatives:

$s=\left[\begin{array}{lllllll}t_{x} & \dot{t}_{x} & t_{y} & \dot{t}_{y} & t_{z} & \dot{t}_{z} & \alpha \dot{\alpha} \beta \dot{\beta} \gamma \dot{\gamma}\end{array}\right]^{T}$

where $t_{x}, t_{y}$, and $t_{z}$ are the translations in the directions of coordinate axes emerging from the center of a reference camera atop the mobile robot. The rotation angles around these axes are: $\alpha, \beta$, and $\gamma$ respectively. The dotted parameters represent the derivatives with respect to time.

The plant equation relates the current state space vector of equation (1) to the plant noise and to the previous state space vector:

$s_{j}=A s_{j-1}+n_{j}$

where $s_{j}$ and $s_{j-1}$ are the state space vectors at frame $j$, the current frame, and at frame $j-1$, the previous frame, $A$ is a $12 \times 12$ matrix relating them according to a uniform speed model, and $n_{j}$ is the plant noise at the current frame assumed to be of normal distribution.

On the other hand, the measurement equation relates the 2-D locations of tracked features to their corresponding camera coordinates functions, and to the measurement noise assumed to have a normal distribution as well:

$I_{j}=f\left(P_{i j k}\right)+\eta_{j}$

where $I_{j}$ is the measurement vector containing the 2-D pixel locations of the features across the stereo cameras, $f\left(P_{i j k}\right)$ is 
a function of the camera coordinates and intrinsic parameters (e.g. focal lengths, and image centers), $i j k$ refers respectively to the feature number, frame number, and camera number (either camera 1 or camera 2 of the stereo pair), and $\eta_{j}$ is the measurement noise. The Jacobian of the EKF is composed of the derivatives of $f\left(P_{i j k}\right)$ with respect to the six pose parameters of equation (1). More about the EKF implementation can be found in [5], [6], and [7].

The overall rotation resulting from the sequence of rotation angles $\alpha, \beta$, and $\gamma$ is given by the rotation matrix: $R$

$=\left[\begin{array}{ccc}\cos \beta \cos \gamma & \sin \alpha \sin \beta \cos \gamma-\cos \alpha \sin \gamma & \cos \alpha \sin \beta \cos \gamma+\sin \alpha \sin \gamma \\ \cos \beta \sin \gamma & \sin \alpha \sin \beta \sin \gamma+\cos \alpha \cos \gamma & \cos \alpha \sin \beta \sin \gamma-\sin \alpha \cos \gamma \\ -\sin \beta & \sin \alpha \cos \beta & \cos \alpha \cos \beta\end{array}\right]$

It is noticed that $\beta$ is the only angle present at every element of the rotation matrix. The reason is that the overall rotation is the result of multiplication of three matrices. Each matrix of the three represents an individual angle having that representing $\beta$ in the middle (since it occurs around the $\mathrm{Y}$ axis, it is inserted between that occur around the $\mathrm{X}$ and $\mathrm{Z}$ axes). This is also true for the translations in the directions of these right-handed coordinate axes. Accordingly, there are singularities when $\beta= \pm \pi / 2$. For example:

$R\left(\beta=\frac{\pi}{2}\right)=\left[\begin{array}{ccc}0 & \sin (\alpha-\gamma) & \cos (\alpha-\gamma) \\ 0 & \cos (\alpha-\gamma) & -\sin (\alpha-\gamma) \\ -1 & 0 & 0\end{array}\right]$

Which means that we can obtain neither $\alpha$ or $\gamma$, but only their difference from the overall rotation matrix.

\subsection{Orientation of Coordinate Systems}

As shown in Figure 1: (A) the coordinate system used in [9] with the directions of its angles of rotations. This coordinate system has the directions of its $\mathrm{X}$ and $\mathrm{Y}$ axes corresponding to that of the image (see Figure $1(\mathrm{~F})$ ). The advantage is having positive focal lengths in the matrix of intrinsic parameters. However, the angle $\beta$ of this coordinate system coincides with the directions of robot allowed rotations. Therefore singularities may be encountered. (B) The suggested coordinate system to overcome the singularity. The reason for this is having the angle $\alpha$ (not $\beta$ ) corresponding to the robot allowed rotations. This comes at the expense of negating the focal length in the horizontal direction of the camera. (C) A coordinate system which is likely to encounter singularities like (A). (D) A coordinate system that overcomes singularity as (B). (E) The coordinate system used in [10]. This is (contrary to the previous ones) a left-handed which can be dealt with by negating the depth $(\mathrm{Z}$ coordinate). However, it is likely to encounter singularities for the same reason as (A), and (C). (F) The robot platform with a camera attached showing the camera coordinates and allowed directions of robot rotations. Any shaking due to an uneven terrain would only produce vibrations of small magnitudes in the two other directions of rotations.

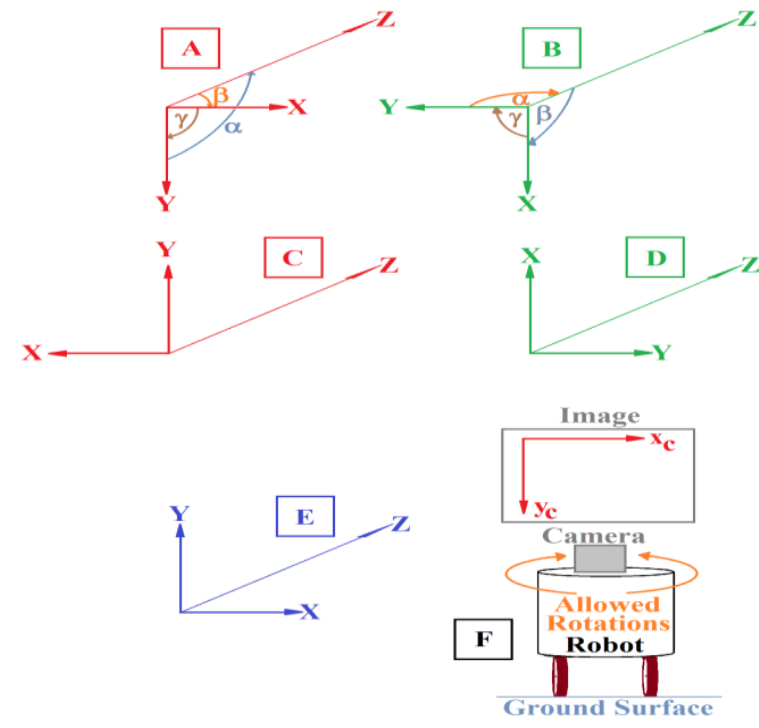

Fig 1: Orientation of coordinate systems, camera coordinates, and angles of rotations

The coordinate systems of (C), (D), and (E) are included only for the sake of completeness. From now on, only the coordinates of (A), and (B) will be considered which are adequate for explaining the rationale.

\section{EXPERIMENTS}

\subsection{Simulations}

A robot carrying eight cameras forming four stereo pairs moved with random translations $\left(t_{x}, t_{y}\right.$, and $\left.t_{z}\right)$ and random rotation angles $(\alpha, \beta$, and $\gamma)$ in the directions of and around the $\mathrm{X}, \mathrm{Y}$, and $\mathrm{Z}$ axes respectively. The translations were taken randomly from \pm 0.005 to \pm 0.025 meter, and the rotation angles were taken randomly from \pm 0.005 to \pm 0.03 radian. The first stereo pair was oriented initially parallel to the reference coordinate system with all angles of rotations equal to zero. The second pair had initially the angle $\alpha$ equal to $\pi / 2$. The third pair had initially the angle $\beta$ equal to $\pi / 2$. The last pair had initially the angle $\gamma$ equal to $\pi / 2$. All cameras had a $6 \mathrm{mms}$ focal length, and a $640 \times 480$ resolution. The baseline between each stereo pair was 0.1 meter. A random noise was added to the 2-D measurements with a normal distribution of zero mean and a 0.5 pixel standard deviation. The motion took place inside a spherical shell with one meter outer radius and $2 / 3$ meter inner radius. The spherical shell was centered at the origin of the coordinate axes. Twenty thousand feature points were distributed randomly within. A sequence of 100 frames was taken simultaneously by each pair. We compared the pose obtained by each stereo pair. The comparison was carried out 500 times under a motion pattern composed from mixed rotation and translation. Table 1 shows the average of absolute errors for the six pose parameters in each case. All absolute errors are given in (meter/radian).

Table 1. Average absolute errors of pose (simulation)

\begin{tabular}{|l|c|c|c|c|c|c|}
\hline Initial & $\boldsymbol{t}_{\boldsymbol{x}}$ & $\boldsymbol{t}_{\boldsymbol{y}}$ & $\boldsymbol{t}_{\boldsymbol{z}}$ & $\boldsymbol{\alpha}$ & $\boldsymbol{\beta}$ & $\boldsymbol{\gamma}$ \\
\hline 0 angles & 0.020 & 0.008 & 0.006 & 0.008 & 0.020 & 0.003 \\
\hline$\alpha=\pi / 2$ & 0.003 & 0.007 & 0.013 & 0.014 & 0.003 & 0.003 \\
\hline$\beta=\pi / 2$ & $1.1 \mathrm{e} 5$ & $1.9 \mathrm{e} 5$ & $1.3 \mathrm{e} 5$ & $2.0 \mathrm{e} 4$ & $1.3 \mathrm{e} 4$ & $1.7 \mathrm{e} 4$ \\
\hline$\gamma=\pi / 2$ & 0.070 & 0.067 & 0.065 & 0.081 & 0.078 & 0.086 \\
\hline
\end{tabular}




\subsection{Real Experiments}

A mobile robot had a stereo pair of two calibrated Canon PowerShot G9 cameras aboard. The cameras had a resolution of $1600 \times 1200$. The baseline between them was $0.14 \mathrm{~m}$. Four sequences of about 200 frames were taken simultaneously by each camera in an ordinary lab scene. The sequences were captured under: pure translation, mixed rotation and translation, pure rotation, and curve motion patterns. The mobile robot and cameras along with samples of the sequence are shown in Figure 2. The coordinate systems (A) and (B) of Figure 1 and subsection 2.2 are used for obtaining the pose parameters under the different motion patterns and compared with the ground truth in in Figure 3, Figure 4, Figure 5, and Figure 6.

\section{DISCUSSIONS}

For the simulations, it is obvious from Table 1 that around the $(\beta=\pi / 2)$, the singularity is devastating. The performances around zero angles of rotation and around $(\alpha=\pi / 2)$ are accurate and close to each other. On the other hand, the performance around $(\gamma=\pi / 2)$ is slightly degraded. The reason for this is that each camera of the stereo pair has its short side (480 pixels) attached to the robot while its long side (640 pixels) erected upright. In this setting, the corresponding features of the stereo pair are mostly distributed along the vertical direction within narrow horizontal band for each camera. Therefore, an approximate small aperture effect appears causing such slight degradation.

For the real experiments, both coordinate systems (A), and (B) nearly coincide for the pure translation pattern as shown in Figure 3. The same is true for the mixed rotation and translation pattern of Figure 4 since the range of rotations are small. As the range increases for the pure rotation pattern (Figure 5), and the curve pattern (Figure 6), coordinate system (A) diverges since its $\beta$ angle corresponds to the direction of robot rotation (see Figure 1). In contrast, coordinate system (B) remains close to the ground truth since its angle $\alpha$ is that varies with the robot rotation. Accordingly, the differences of coordinate systems are taken into considerations in the figures. For example, both $\beta$ of coordinate system (A), and $-\alpha$ of coordinate system (B) are compared to $\beta$ of the ground truth (robot motion obtained from the computer steering it). The corresponding pose parameters between coordinate systems (A), and (B) are mentioned in figure captions. They are also clear from Figure 1.There are three main reasons for the singularity which causes the divergence of pose estimation approaches. The first (and the less probable) is having the angle $\beta$ exactly equal to $\pm \pi / 2$. The second is having the angle $\beta$ in the vicinity of $\pm \pi / 2$ (i.e. within 0.2 rad from either side). This situation is not a precise singularity from the mathematical point of view. However, the two other estimated angles $(\alpha$, and $\gamma$ ) suffer from abrupt changes from frame to frame due to any small perturbation in $\beta$. The third is the derivative calculations (e.g. within the EKF Jacobian). The angle $\beta$ is present in all entries of the rotation matrix (see equation (4)). Therefore, the instability due to the derivative taking is exaggerated as the magnitude of $\beta$ increases (even to \pm 0.4 or $\pm 0.5 \mathrm{rad}$ which is far less than $\pm \pi / 2$, see Figure 5).

\section{CONCLUSIONS}

An approach is proposed to overcome the singularity of the popular and physically tangible Euler angles rotation representation. The reference coordinate system is rotated to have the angle $\alpha$ corresponding to the robot direction of rotation. In this way, the variation of the angle $\beta$ is kept within a small magnitude due to the robot shaking during its motion. Therefore, the causes of singularity and mathematical instability are avoided. For the future work, overcoming the Euler angles singularities for flying objects such as the quadcopters will be studied.

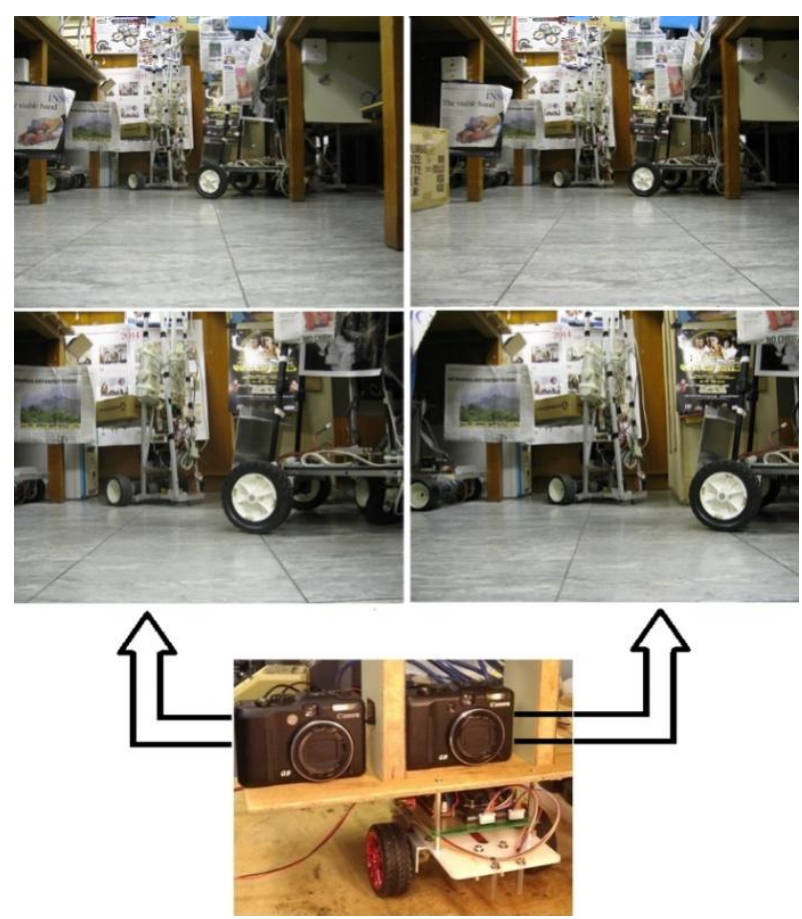

Fig 2: Robot, stereo pair, and samples of the sequences

\section{REFERENCES}

[1] N. Elfadil, S. A. Aljahdali, and M. Moawad, "System for communicating a vehicle position and speed during accident", International Journal of Computer Applications ,Volume 94 , No. 19, (May 2014), 13-18

[2] A.B.M. K. Hassan, and E. Zahir," Optimizing the turning velocity in a line follower robot", International Journal of Computer Applications, Volume 117, No. 3, ( May 2015), 14-17

[3] I. Sa, and H. S. Ahn, "Visual 3D model-based tracking toward autonomous live sports broadcasting using a VTOL unmanned aerial vehicle in GPS-impaired environments", International Journal of Computer Applications, Volume 122, No. 7, (July 2015), 1-7

[4] Yang, M., Yu, Q., Wang H., and Zhang B. 2004. Vision based real-time pose estimation for intelligent vehicles. In Proceedings of the Intelligent Vehicle Symposium.

[5] A. Chiuso, P. Favaro, H. Jain, and S. Soatto, "Structure from motion causally integrated over time", IEEETransactions on Pattern Recognition and Machine Intelligence, Volume 24, No. 4, (April 2002), 523-535.

[6] A. Azarbayejani, and A. P. Pentland, "Recursive estimation of motion, structure, and focal length", IEEE Transactions on Pattern Recognition and Machine Intelligence, Volume 17, No. 6, (June 1995), 562-575.

[7] Lippiello, V., Sciliano, B., and Villani, L. 2001. Position and orientation estimation based on Kalman filtering of stereo images. In Proceedings of the IEEE International Conference on Control Applications 
[8] M. E. Ragab, K.H. Wong, "Rotation within camera projection matrix using Euler angles, quaternions and angle-axes", ACTA Press International Journal of Robotics and Automation, Volume 24, No. 4, (Dec. 2009), 312-318.

\section{APPENDIX}
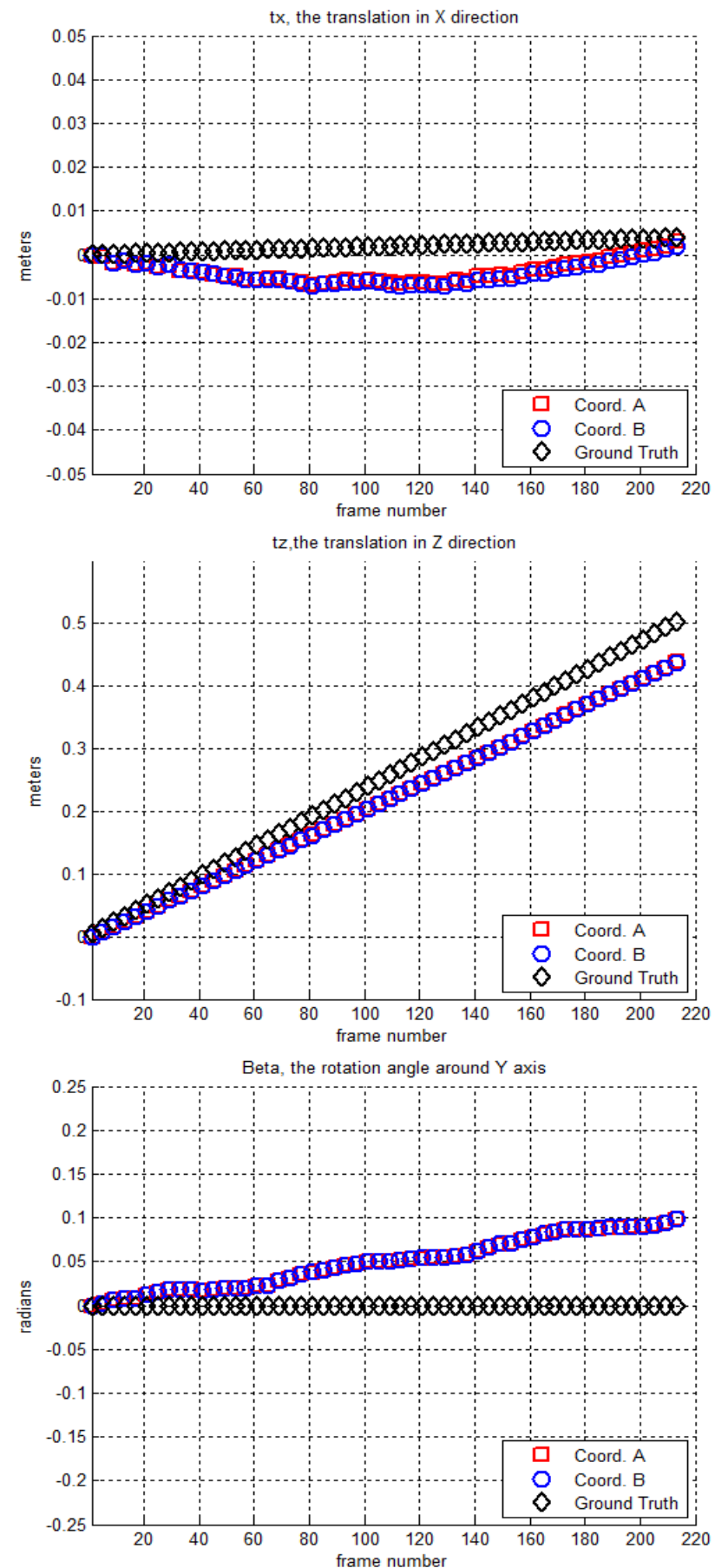

[9] Faugeras, O. and Luong, Q. T. 2001 The Geometry of Multiple Images. MIT Press.

[10] Hartley, R. and Zisserman, A. 2003 Multiple View Geometry in Computer Vision. Cambridge University Press.
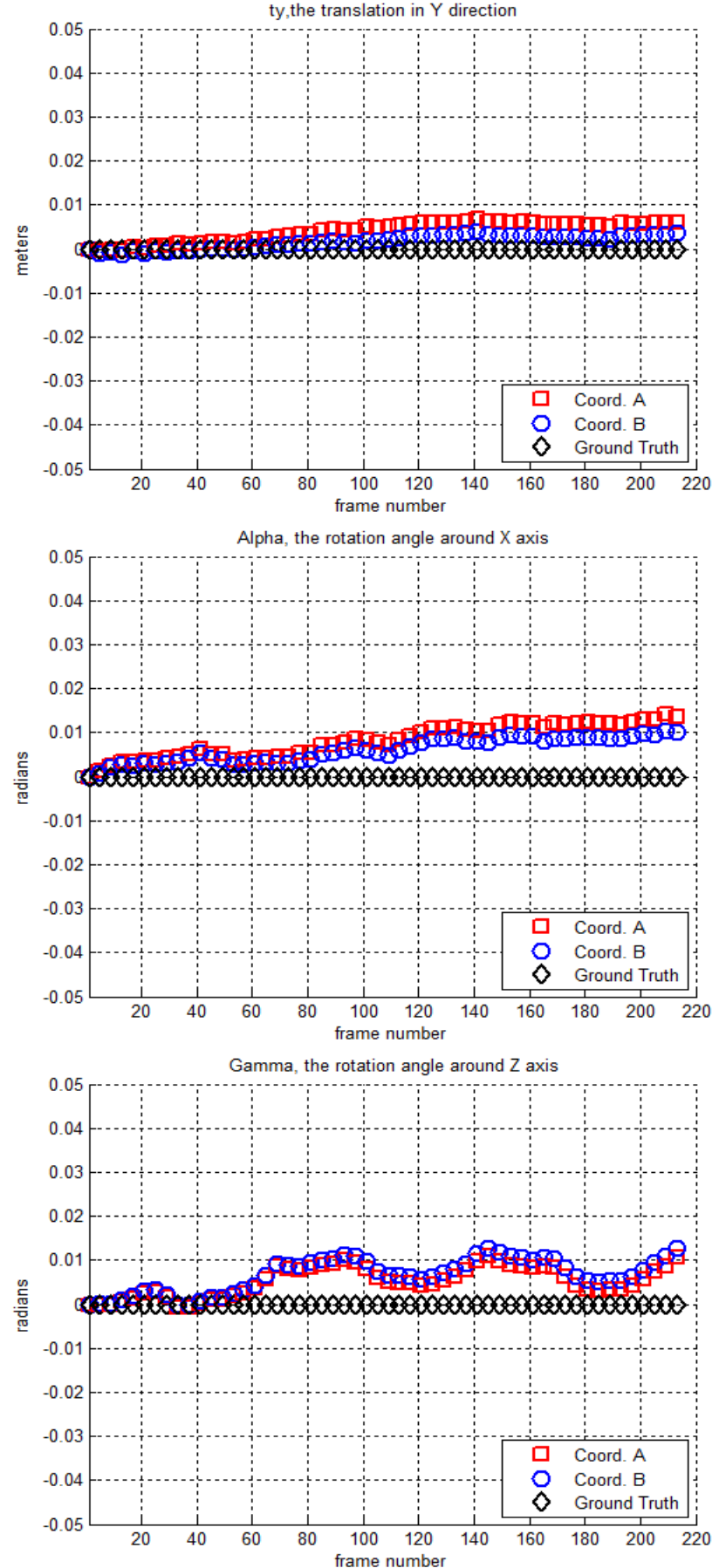

Fig 3: Real experiment (pure translation), Coord. A, and Coord. B are the coordinate systems (A), and (B) of Figure 1. It is noted that: $\left(t_{x}, t_{y}, t_{z}, \alpha, \beta\right.$, and $\left.\gamma\right)$ of Coord. A correspond respectively to $\left(-t_{y}, t_{x}, t_{z},-\beta, \alpha\right.$, and $\left.\gamma\right)$ of Coord. B. Coord. A and Coord. B nearly coincide for this sequence 

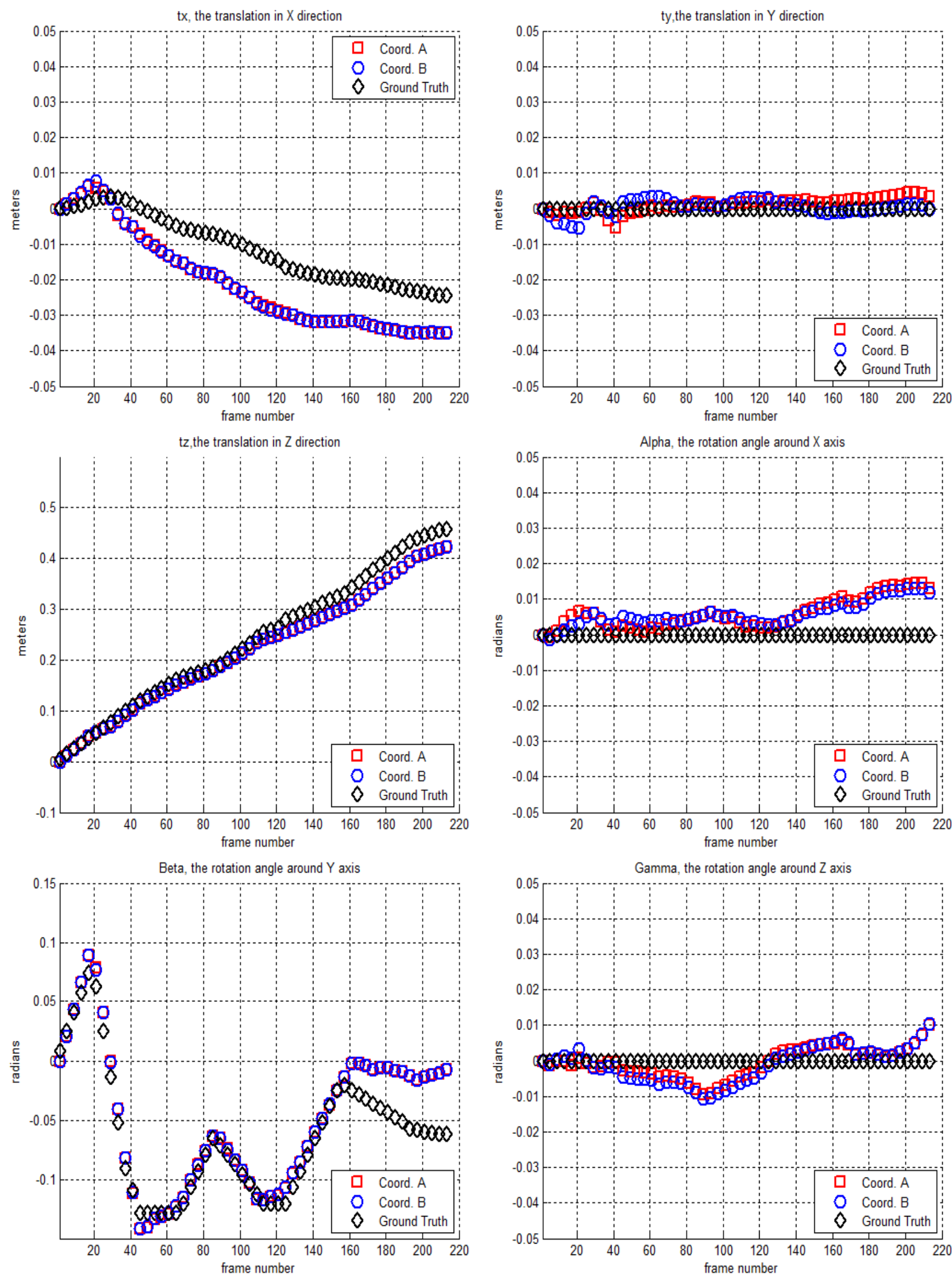

Fig 4: Real experiment (mixed rotation and translation), Coord. A, and Coord. B are the coordinate systems (A), and (B) of Figure 1. It is noted that: $\left(t_{x}, t_{y}, t_{z}, \alpha, \beta\right.$, and $\left.\gamma\right)$ of Coord. A correspond respectively to $\left(-t_{y}, t_{x}, t_{z},-\beta, \alpha\right.$, and $\left.\gamma\right)$ of Coord. B. Coord. A and Coord. B nearly coincide for this sequence 

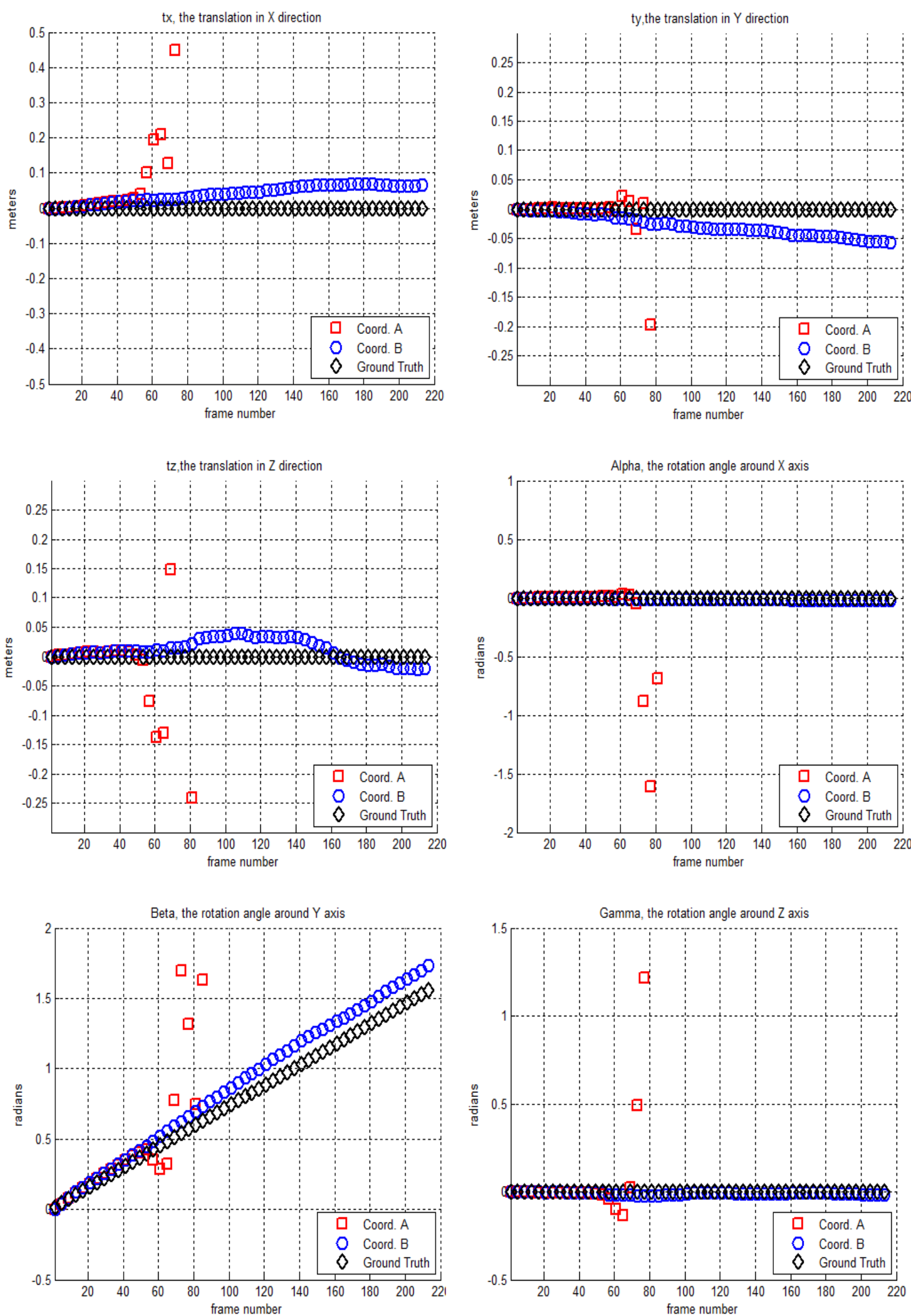

Fig 5: Real experiment (pure rotation), Coord. A, and Coord. B are the coordinate systems (A), and (B) of Figure 1. It is noted that: $\left(t_{x}, t_{y}, t_{z}, \alpha, \beta\right.$, and $\left.\gamma\right)$ of Coord. A correspond respectively to $\left(-t_{y}, t_{x}, t_{z},-\beta, \alpha\right.$, and $\left.\gamma\right)$ of Coord. B. Coord. A diverges while Coord. B nearly coincides with the ground truth for this sequence 

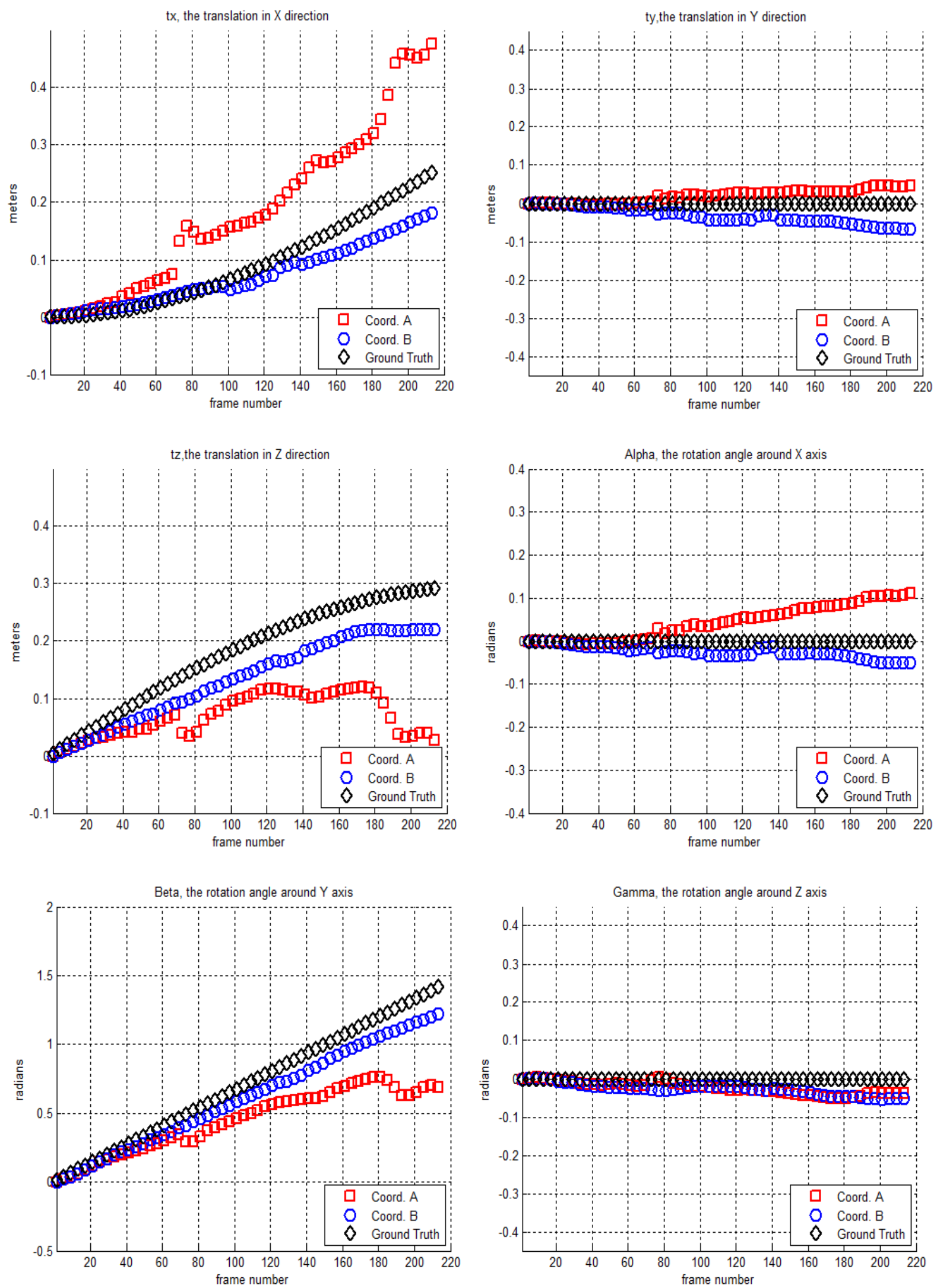

Fig 6: Real experiment (curve), Coord. A , and Coord. B are the coordinate systems (A), and (B) of Figure 1. It is noted that: $\left(t_{x}, t_{y}, t_{z}, \alpha, \beta\right.$, and $\left.\gamma\right)$ of Coord. A correspond respectively to $\left(-t_{y}, t_{x}, t_{z},-\beta, \alpha\right.$, and $\left.\gamma\right)$ of Coord. B. Coord. A diverges while Coord. $B$ remains close to the ground truth for this sequence. 